\title{
Flow responses alteration by geometrical effects of tubercles on plates under the maximal angle of attack
}

Proc IMechE Part C:

J Mechanical Engineering Science 2021, Vol. 235(19) 3975-3987 (C) IMechE 2020 Article reuse guidelines: sagepub.com/journals-permissions DOI: 10.1 I 77/0954406220975434 journals.sagepub.com/home/pic

(SSAGE

\author{
KS Mu', ABH Kueh ${ }^{2} \mathbb{D}$, PN Shek', MR Mohd Haniffah ${ }^{3}$ and \\ BC Tan'
}

\begin{abstract}
Plates with leading-edge tubercles experience beneficially more gradual aerodynamics stalling when entering the poststall regime. Little is known, however, about the corresponding aquatic flow responses when these tubercles-furnished plates are subjected to the maximal angle of attack, with the flow direction perpendicular to their planar area. Hence, this study presents numerically, by means of the flow behavior solver ANSYS, the flow responses alteration in terms of the geometrical effects of tubercles on plates through changes in amplitudes $(5 \mathrm{~mm}, 10 \mathrm{~mm}, 15 \mathrm{~mm})$ and wavelengths $(50 \mathrm{~mm}, 100 \mathrm{~mm}, 150 \mathrm{~mm})$ under the maximal angle of attack in comparison to a control case, i.e., without tubercles. Additional to the commonly examined flow velocity and pressure, characteristics such as wake (area, reattachment length, flow recirculation intensity) and newly defined downstream vortical parameters (area, perimeter, and Feret diameters) for the vortex region have been proposed and assessed. It is found that the drag increases with the tubercle wavelength but corresponds inversely with the tubercle amplitude. By correlating with the best beneficial velocity and pressure profiles, it has been characterized that the optimally performing plate is the one that generates the greatest flow recirculation intensity, wake area, and reattachment length, corresponding to the capability to produce also the highest vortical area, perimeter, and major Feret diameter. Compared to the control case, all plates with tubercles alter beneficially these flow behaviors. In conclusion, plates with tubercles contribute favorably to the flow behaviors under the maximal angle of attack compared to the control case while the newly proposed downstream parameters could serve capably as alternatives in corroborating the flow physics description in future studies.
\end{abstract}

\section{Keywords}

Geometrical effect, tubercle, maximal angle of attack, vortical property, Feret diameter

Date received: 18 May 2020; accepted: 31 October 2020

\section{Introduction}

In the past several years, studies were actively conducted in numerous aspects to enhance the efficiency of both wind and hydro turbines. ${ }^{1,2}$ One of the challenges for turbine designers is the appropriate profile and geometrical designs of the turbine blade. ${ }^{2-5}$ Thicker blade sections are needed to satisfy the structural integrity and to prevent cavitation. However, this strategy increases the unfavorable drag. Also, keeping a consistent output is difficult especially for turbines operating at unsteady flow conditions. Therefore, an efficient blade design in terms of maximizing turbine rotation with minimum drag for greater energy output through geometrical variation has been a continually explored research subject by the energy harvesting communities. Recently, researchers seeking for cheap, clean, and sustainable energy solutions have ventured and found valuable designs available in nature for inspiration and innovation. ${ }^{6-8}$ This investigation trend allows the employment of designs inspired by nature as templates for man-made structures. One of the current bio-inspired innovations involves the application of a series of sinusoidally distributed tubercles on the turbine blade for an enhanced energy harvesting, a concept

\footnotetext{
'Construction Research Centre, Faculty of Engineering, Universiti Teknologi Malaysia, Johor, Malaysia

${ }^{2}$ Department of Civil Engineering, Faculty of Engineering, Universiti Malaysia Sarawak, Sarawak, Malaysia

${ }^{3}$ Center for Coastal and Ocean Engineering, Faculty of Engineering, Universiti Teknologi Malaysia, Johor, Malaysia

Corresponding author:

$\mathrm{ABH}$ Kueh, Department of Civil Engineering, Faculty of Engineering, Universiti Malaysia Sarawak, 94300 Kota Samarahan, Sarawak, Malaysia. Email: kbhahmad@unimas.my
} 